\title{
Optimal approximation of fractional derivatives through discrete-time fractions using genetic algorithms
}

\author{
J.A. Tenreiro Machado, Alexandra M. Galhano, Anabela M. Oliveira, József K. Tar
}

ABSTRACT

This study addresses the optimization of rational fraction approximations for the discrete- time calculation of fractional derivatives. The article starts by analyzing the standard techniques based on Taylor series and Padé expansions. In a second phase the paper re-evaluates the problem in an optimization perspective by tacking advantage of the flex-ibility of the genetic algorithms.

Keywords

Fractional derivatives, Fractional calculus, Optimization, Genetic algorithms

\section{Introduction}

Fractional calculus (FC) deals with the generalization of integrals and derivatives to a non-integer order and, in the last decades, its application verified a large development in the areas of physics and engineering.

The fundamental aspects of the FC theory and the study of its properties can be addressed in Refs. [1-4]. A significant progress occurred in the application of the FC concepts and we can mention a large volume of research about viscoelasticity, biology, electronics, signal processing, diffusion and wave propagation, modeling and control [5-14]. Nevertheless, FC is still considered an 'exotic' mathematical tool and its adoption requires some efforts towards the development of clear algorithms.

One of the reasons for this state of affairs is the complexity of the algorithms involved in the calculation of fractional derivatives. The generalization of the integrodifferential operator requires the adoption of approximations of irrational functions through series or rational fraction expansions [15-22]. While the main volume of contributions has focused in getting expansion schemes, the problem of a systematic optimization procedure was not yet tackled.

In this line of thought, this paper addresses the optimal calculation of fractional order expressions and is organized as follows. Section 2 introduces the calculation of fractional derivatives and formulates the problem of fraction approximation of irrational formulae through genetic algorithms. Section 3 presents a set of experiments that demonstrate the effectiveness of the proposed optimization method. Finally, Section 4 outlines the main conclusions. 



\section{Problem formulation and adopted tools}

This section introduces the main mathematical concepts and algorithms used in the rest of the article. Section 2.1 presents the definition of fractional derivative adopted together with the discretization rules for real time calculation. Section 2.2 outlines the fundamental aspects underlying the GA optimization scheme.

\subsection{Fractional order expressions}

Since the foundation of the differential calculus the generalization of the concept of derivative and integral to a noninteger order $a$ has been the subject of several approaches such as the Riemann-Liouville, the Grünwald-Letnikov, the Caputo and the Fourier/Laplace definitions.

The Grünwald-Letnikov definition of a derivative of fractional order $a$ of the signal $x(t), D^{a} x(t)$, is given by:

$$
D^{\alpha} x(t)=\lim _{h \rightarrow 0} \frac{1}{h^{\alpha}} \sum_{k=0}^{\infty} \frac{(-1)^{k} \Gamma(\alpha+1) x(t-k h)}{\Gamma(k+1) \Gamma(\alpha-k+1)}
$$

where $C$ is the gamma function and $h$ is the time increment. This formulation inspires a discrete-time calculation algorithm, based on the approximation of the time increment $h$ through the sampling period T, yielding the equation in the $z$ domain:

$$
\begin{aligned}
& Z\left\{D^{\alpha} x(t)\right\} \approx\left[\frac{1}{T^{\alpha}} \sum_{k=0}^{\infty} \frac{(-1)^{k} \Gamma(\alpha+1)}{k ! \Gamma(\alpha-k+1)} z^{-k}\right] X(z) \\
& \geq X(z)=Z\{x(t)\} .
\end{aligned}
$$

The implementation of expression (2) corresponds to an $r$-term truncated series given by:

$$
Z\left\{D^{\alpha} x(t)\right\} \approx\left[\frac{1}{T^{\alpha}} \sum_{k=0}^{r} \frac{(-1)^{k} \Gamma(\alpha+1)}{k ! \Gamma(\alpha-k+1)} z^{-k}\right] X(z)
$$

Expression (2) represents the Euler (or first backward difference) approximation in the so-called $s$ ? $z$ conversion scheme. Another possibility, often adopted in control system design, consists in the Tustin (or bilinear) rule. The Euler and Tustin rational expressions, $H_{0} \partial z^{-1} \mathrm{P} 1 / 4 \frac{1}{T} \delta 1-z^{-1} \mathrm{p}$ and $H_{1} \partial z^{-1 \mathrm{P}} 1_{4}^{1 / 4} \frac{2}{T} \frac{1-z^{-1}}{1 \mathrm{p} z^{-1}}$, are often called generating approximants of zero and first order, respectively. Therefore, the generalization of these conversion methods leads to the non-integer order $a$ results:

$$
\begin{aligned}
& s^{\alpha} \approx\left[\frac{1}{T}\left(1-z^{-1}\right)\right]^{\alpha}=H_{0}^{\alpha}\left(z^{-1}\right) \\
& s^{\alpha} \approx\left(\frac{2}{T} \frac{1-z^{-1}}{1+z^{-1}}\right)^{\alpha}=H_{1}^{\alpha}\left(z^{-1}\right)
\end{aligned}
$$

We can obtain a family of fractional differentiators generated by $H_{0}^{a}\left(z^{-1}\right)$ and $H_{1}^{a} \partial z^{-1} p$ weighted by the factors $p$ and $1-p$, yielding:

$$
H_{a v}\left[z^{-1} ;(p, \alpha)\right]=p H_{0}^{\alpha}\left(z^{-1}\right)+(1-p) H_{1}^{\alpha}\left(z^{-1}\right)
$$

In order to get a rational expression, the final approximation corresponds to a truncated Taylor series or a rational fraction expansion. Due to its superior performance often it is used a fraction:

$$
H_{k}\left(z^{-1}\right)=\frac{\sum_{i-0}^{k} a_{i} z^{-i}}{\sum_{i=0}^{k} b_{i} z^{-i}}, \quad a_{i}, b_{i} \in \Re
$$

where $k 2 @$ denotes the order of the approximation. Moreover, usually it is adopted a Padé expansion in the neighborhood of $z^{-1}=0$ and, since one parameter is linearly dependent, it is established $b_{0}=1$.

\subsection{Optimization through genetic algorithms}

A GA is a computational technique to find exact or approximate solutions to optimization and search problems [23-25]. GAs are simulated in a computing system, and consist in a population of representations of candidate solutions, of an optimization problem, that evolve toward better solutions. 
Once the genetic representation and the fitness function are defined, the GA proceeds to initialize a population of solutions randomly, and then to improve it through the repetitive application of mutation, crossover and selection operators.

The evolution usually starts from a population of randomly generated individuals. In each generation, not only the fitness of every individualin the population isevaluated, butalso severalindividuals arestochastically selected from thecurrent population and modified to form a new population. Thenew population is then used in the next iteration of the algorithm. The GA terminates when either the maximum number of generations $N$ is produced, or a satisfactory fitness level has been reached.

During the successive generation, a part or the totality of the population is selected to breed a new generation. Individual solutions are selected through a fitness-based process, where fitter solutions (measured by a fitness function) are usually more likely to be selected. The pseudo-code of the GA is:

1. Choose the initial population

2. Evaluate the fitness of each individual in the population

3. Repeat

3.1. Select best-ranking individuals to reproduce

3.2. Breed new generation through crossover and mutation and give birth to offspring

3.3. Evaluate the fitness of the offspring individuals

3.4. Replace the worst ranked part of population with offspring

4. Until termination

The present article adopts also the common technique of elitism, which is the process of selecting the better individuals to form the parents in the offspring generation.

\section{Fractional order differentiation}

In this section we study the fraction approximation for the calculation of fractional expressions. In Section 3.1 we analyze the properties of Padé fractions and, in Section 3.2, we formulate a new optimization method based in a GA scheme.

\subsection{Padé fraction expansions}

Bearing in mind expressions (3-6) we examine the approximation of $D^{a}, a=1 / 2$, when $p=3 / 4$ for Padé fraction expansions of orders $k=\{1,2,3,4\}$. The comparison can be established either in the time, or the frequency domains. Although
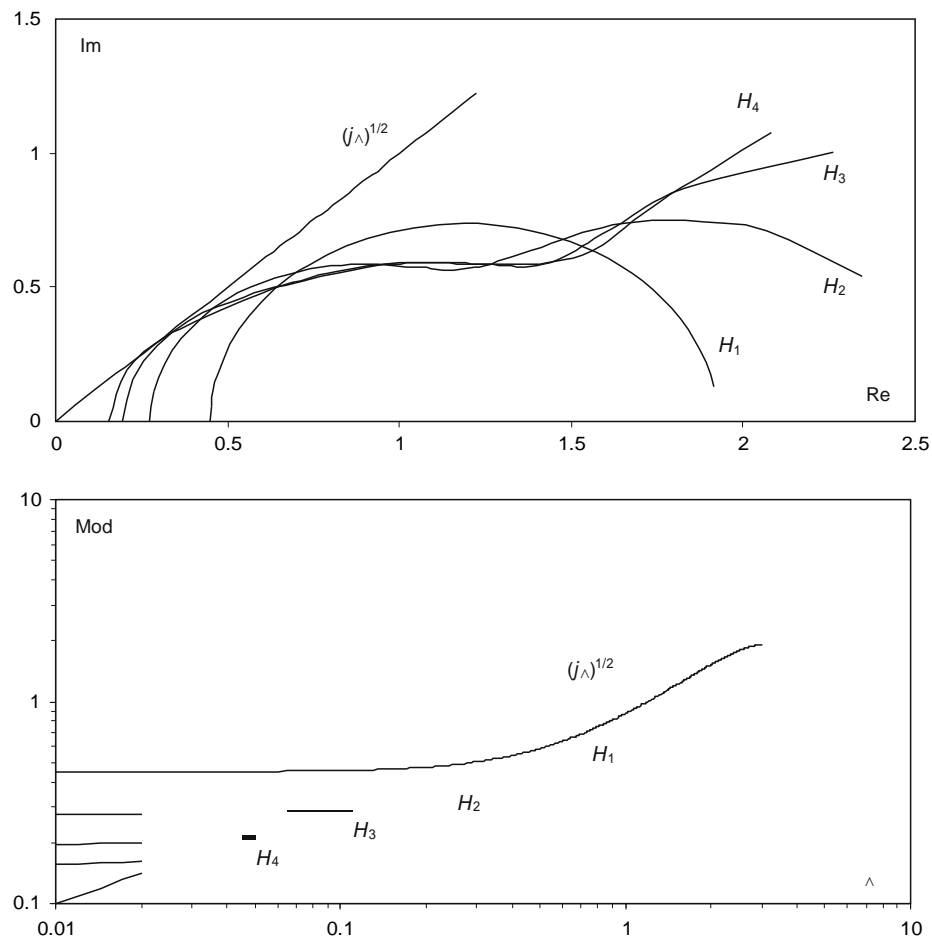

Fig. 1. Polar diagrams and amplitude Bode diagrams of $D^{1 / 2}$ Padé fraction expansions $H_{k}\left(z^{-1}\right), k=\{1,2,3,4\}$, based on the expressions 5,6 , with $p=3 / 4, v e r s u s$ the ideal case $H_{d}(j X)=(j X)^{1 / 2}, 06 X<3 \mathrm{rads}^{-1}, T=1$.

conceptually equivalent, for the purpose of defining a optimization criteria simple to implement in the AG fitness function, in this paper it is adopted the frequency response. Therefore, for the frequency-based expressions is considered the transfor- 


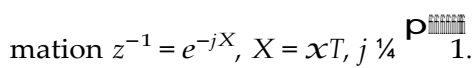

Fig. 1 depicts the polar and amplitude Bode diagrams versus the ideal plot for $T=1$. We observe that the charts of $H_{k}$, $k=\{1,2,3,4\}$, are of the same type, but vary with the order $k$ of the approximation. The frequency response is not the optimization objective since the criteria for the fraction expansion is simply the approximation in the neighborhood of $z^{-1}=0$. Therefore, we conclude that the problem is ill posed and that it should be clearly formulated as an optimization process.

\subsection{Fraction optimization with genetic algorithms}

In this section we develop the GA optimization of rational fraction approximation to fractional order expressions.

The GA population, with $P$ individuals, is constituted by a series of candidate fraction coefficients $\frac{1 / 2}{2} a_{1} \cdots a_{k} \quad b_{1} \quad \cdots \quad b_{k} J$, with $b_{0}=1.0$. In the optimization two possible criteria, leading to distinct fitness functions, are studied:

$$
\begin{aligned}
& J_{1}= \begin{cases}\min _{P} \sum_{i=1}^{n}\left\langle\left\{\operatorname{Re}\left[H_{d}\left(\Omega_{i}\right)\right]-\operatorname{Re}\left[H_{k}\left(\Omega_{i}\right)\right]\right\}^{2}+\left\{\operatorname{Im}\left[H_{d}\left(\Omega_{i}\right)\right]-\operatorname{Im}\left[H_{k}\left(\Omega_{i}\right)\right]\right\}^{2}\right\rangle & \text { if } H \text { stable } \\
J_{10} & \text { if } H \text { unstable }\end{cases} \\
& J_{2}= \begin{cases}\min _{P} \sum_{i=1}^{n} \frac{\left\{\operatorname{Re}\left[H_{d}\left(\Omega_{i}\right)\right]-\operatorname{Re}\left[H_{k}\left(\Omega_{i}\right)\right]\right]^{2}+\left[\operatorname{Rm}\left[H_{d}\left(\Omega_{i}\right)\right]-\operatorname{lm}\left[H_{k}\left(\Omega_{i}\right)\right]\right]^{2}}{\left.\left\langle\left(\Omega_{i}\right)\right]+\operatorname{Re}\left[H_{k}\left(\Omega_{i}\right)\right]\right]^{2}+\left[\operatorname{Im}\left[H_{d}\left(\Omega_{i}\right)\right]+\operatorname{Im}\left[H_{k}\left(\Omega_{i}\right)\right]\right]^{2}} & \text { if } H \text { stable } \\
J_{20} & \text { if } H \text { unstable }\end{cases}
\end{aligned}
$$

where $H_{d}\left(X_{i}\right)$ and $H_{k}\left(X_{i}\right)$ denote the desired and the $k$ th-order fraction frequency responses at frequency $X_{i}$, respectively, and $\operatorname{Re}[$ ] and $\operatorname{Im}[$ ] represent the real and imaginary parts. For the fitness evaluation two alternative sets of frequency sampling points, $S_{i}(X)=X_{\min } 6 \times 6 X_{\max }, i=1,2$, are adopted, namely with a linear variation $\left(S_{1}\right)$ and with a logarithmic variation $\left(S_{2}\right)$. Moreover, the evaluation verifies if the transfer function $H_{k}$ is stable, assigning a large value $J_{10}$ or $J_{20}$ for the unstable case, or calculating the deviation between $H_{d}\left(X_{i}\right)$ and $H_{k}\left(X_{i}\right)$, for the stable case.

In what concerns the fitness functions, the expression of $J_{1}$ points to the minimization of the absolute error, while the expression of $J_{2}$ is inspired in the minimization of the relative error. With respect to the frequency sampling sets, $S_{1}$ addresses representations with linear scales, while $S_{2}$ suggests representations with logarithmic scales.

In the experiments, the GA adopts a population of $P=10^{3}$ individuals, mutation probability $p_{m}=10^{-1}$, single point crossover and reproduction within all population considering elitism. The GA terminates when reached $N=10^{5}$ generations. It was verified that the GA converges more easily when the initial population is composed of stable transfer functions. Therefore, the fractions coefficients are generated through a uniform probability distribution function, but all unstable fractions are eliminated, being substituted by new elements, before initiating the GA evolution. It is examined the evaluation of a derivative of order $a=1 / 2$ through the fraction approximations $H_{k}, k=\{1,2,3\}$, with $T=1$. Furthermore, $n=30$ sampling points are adopted in the intervals $S_{1}(X) \quad\left\{X_{\min }, X_{\max }\right\} \quad\{0,3\}[\mathrm{rad} / \mathrm{s}]$ or $S_{2}(X) \quad\left\{X_{\min }, X_{\max }\right\} \quad\left\{10^{-3}, 3\right\}[\mathrm{rad} / \mathrm{s}]$.

Figs. 2-4 depict the frequency response, namely the polar and amplitude Bode diagrams, for all combinations of fitness functions and frequency sampling sets, versus the ideal plot. Tables 1-3 show the coefficients (truncated to five digits) of the corresponding fraction approximations $H_{k}, k=\{1,2,3\}$.

It should be noted that new fitness functions, different sets of sampling points, and other values for the limits of the interval of approximation, lead to distinct fraction coefficients. By other words, the GA produces a particular solution for each distinct optimization formulation and for each different set of restrictions.

The GA has a stochastic nature and, as usual when applying evolutionary algorithms, the results of a given GA execution may correspond to a local minimum, instead of a global minimum. Therefore, it is required a careful check of the final fraction before accepting the results. Nevertheless, several distinct experiments demonstrated that with the present set of numerical parameters the GA-generated results have a small variance.

It was verified that the GA-generated fractions were stable; however, in several cases, it was found that the roots of the denominator were close to the limit condition $\mathrm{j} z \mathrm{j}=1$. If more demanding stability conditions are necessary, it is straightforward to adapt conditions 7,8 for getting a condition of the type $\mathrm{j} z \mathrm{j}=c$, with $0<c 61$.

Analyzing the results we conclude that:

- In general, the higher the order of the fraction, the better the approximation.

- The fitness $J_{1}$ produces superior results for the polar diagram, while $J_{2}$ produces better results for the Bode diagram.

- The frequency sampling set $S_{1}$ seems well adapted to the polar diagram, while $S_{2}$ produces better results for the Bode diagram.

Obviously, the optimization GA requires a larger computational time, the larger the number of sampling points, the higher the number of population elements and the larger the number of coefficients, that is, the higher the order of the fraction approximation. Nevertheless, since the fraction calculation is performed off-line, there is no problem about the computational load and the numerical experiments demonstrated that the algorithm requires common computer resources. 

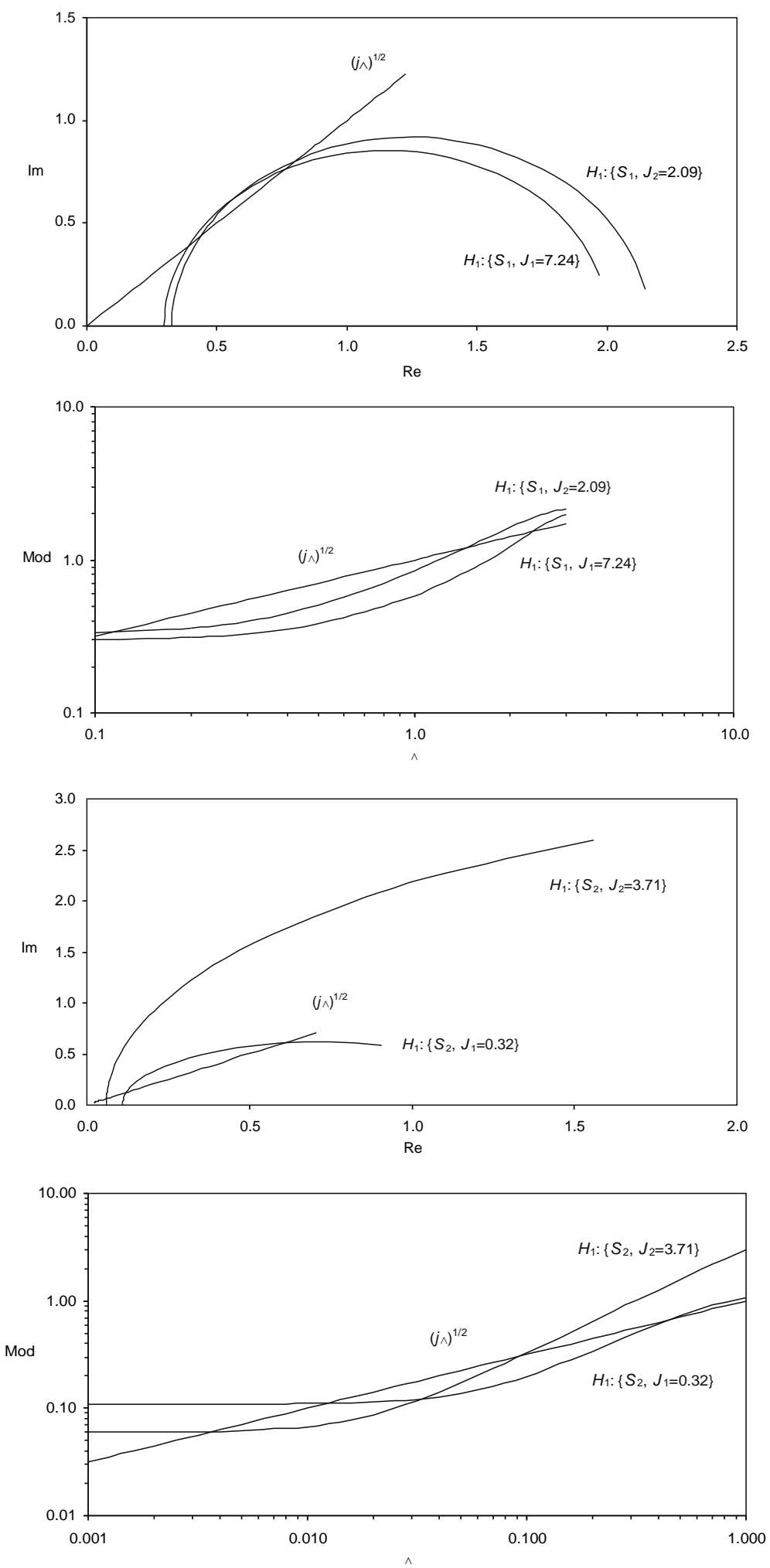

Fig. 2. Polar and amplitude Bode diagrams for approximations $H_{1}$ generated through a GA with finesses $J_{n}, n=\{1,2\}$ and sampling sets $S_{m}, m=\{1,2\}$.

Comparing the Padé expansions and the GA-generated fractions we verify clearly the superior frequency response of the optimization evolutionary scheme. 

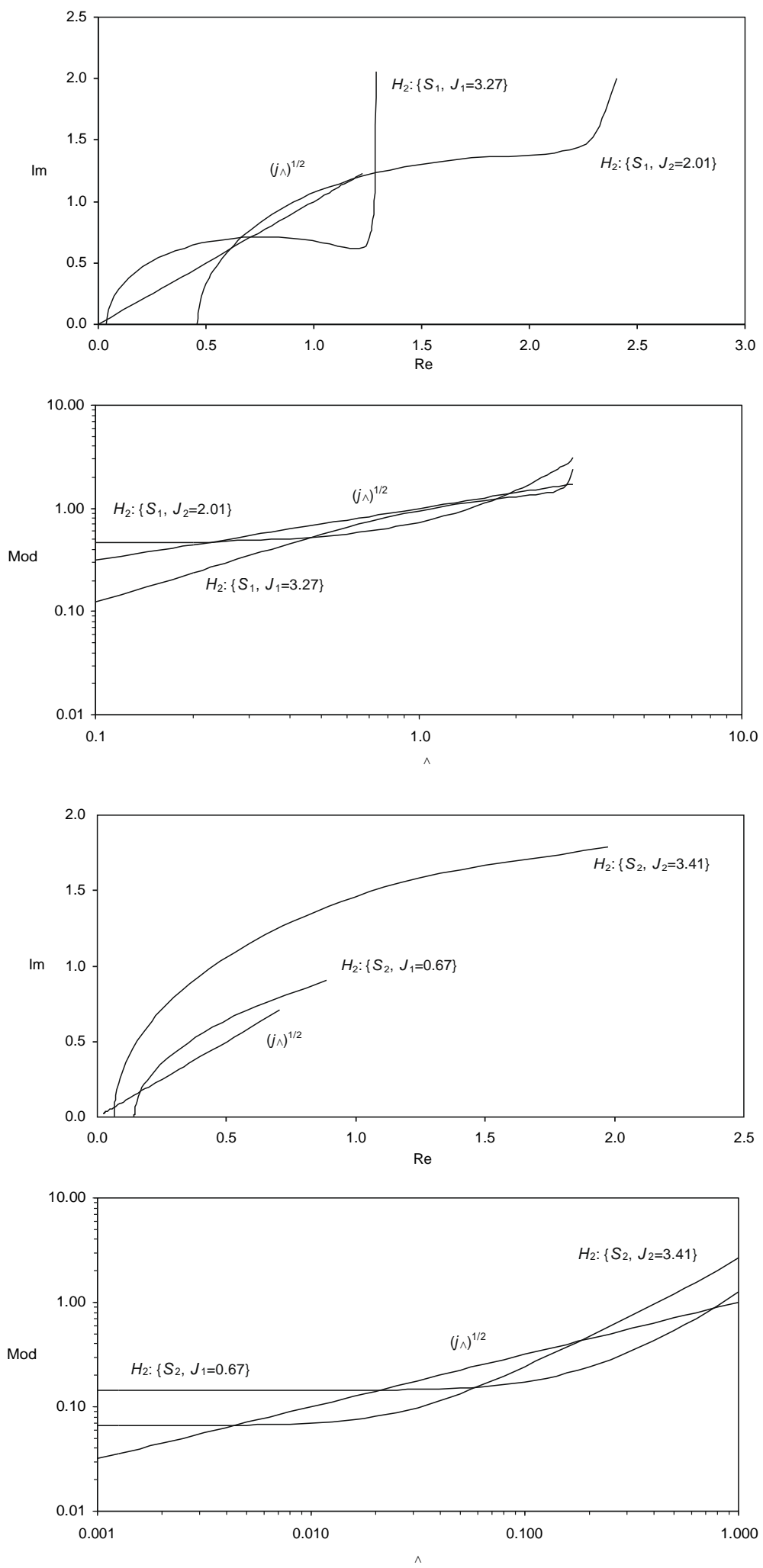

Fig. 3. Polar and amplitude Bode diagrams for approximations $H_{2}$ generated through a GA with finesses $J_{n}, n=\{1,2\}$ and sampling sets $S_{m}, m=\{1,2\}$.

The evolutionary optimization approach is not restricted to the calculation of fractional-order derivatives and integrals. In fact, the scheme can be easily used in the fraction approximation of any fractional expression, being a typical 

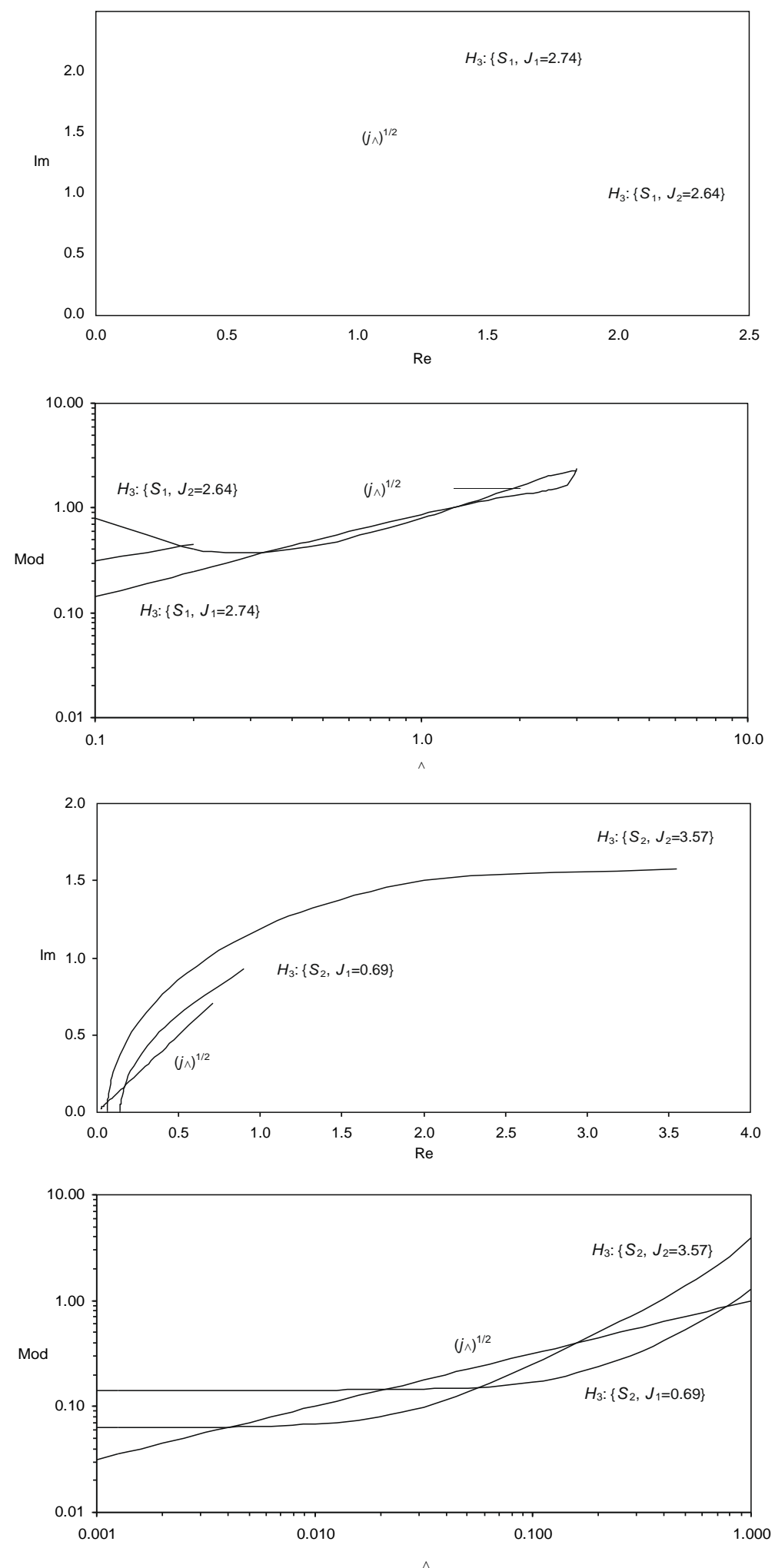

Fig. 4. Polar and amplitude Bode diagrams for approximations $H_{3}$ generated through a GA with finesses $J_{n, n}=\{1,2\}$ and sampling sets $S_{m}, m=\{1,2\}$.

case the fractional $P I^{k} D^{l}$ controller with transfer function $G(s)=K_{p}+K_{i} s^{-k}+K_{d} s^{l}, 0<k, l 61$, where $s$ represents the Laplace variable. 
Table 1

Coefficients of the fraction approximation $H_{1}$ for $D^{1 / 2}$ with finesses $J_{n}, n=\{1,2\}$ and sampling sets $S_{m}, m=\{1,2\}$.

\begin{tabular}{rlllrr}
$n$ & $m$ & $a_{0}$ & $a_{1}$ & $b_{0}$ & $J_{1}$ \\
1 & 1 & 0.85147 & -0.45036 & 1.0 & 0.35111 \\
1 & 2 & 1.09732 & -0.71955 & 1.0 & 0.16026 \\
2 & 1 & 0.98643 & -0.92363 & 1.0 & -0.42581 \\
2 & 2 & 3.14041 & -3.08235 & 1.0 & 0.32000 \\
\hline
\end{tabular}

Table 2

Coefficients of the fraction approximation $H_{2}$ for $D^{1 / 2}$ with finesses $J_{n}, n=\{1,2\}$ and sampling sets $S_{m}, m=\{1,2\}$.

\begin{tabular}{|c|c|c|c|c|c|c|c|c|}
\hline$n$ & $m$ & $a_{0}$ & $a_{1}$ & $a_{2}$ & $b_{0}$ & $b_{1}$ & $b_{2}$ & $J_{n}$ \\
\hline 1 & 1 & 0.97602 & -0.15552 & -0.76935 & 1.0 & 0.72657 & -0.27340 & 3.26990 \\
\hline 1 & 2 & 1.15168 & 0.58865 & -0.42819 & 1.0 & 1.42696 & 0.42697 & 2.00957 \\
\hline 2 & 1 & -0.40260 & 4.50186 & -3.57756 & 1.0 & 1.82223 & 0.82239 & 0.66531 \\
\hline 2 & 2 & 0.29881 & 5.06626 & -5.20914 & 1.0 & 1.19292 & 0.19306 & 3.41291 \\
\hline
\end{tabular}

Table 3

Coefficients of the fraction approximation $H_{3}$ for $D^{1 / 2}$ with finesses $J_{n}, n=\{1,2\}$ and sampling sets $S_{m}, m=\{1,2\}$.

\begin{tabular}{|c|c|c|c|c|c|c|c|c|c|c|}
\hline$n$ & $m$ & $a_{0}$ & $a_{1}$ & $a_{2}$ & $a_{3}$ & $b_{0}$ & $b_{1}$ & $b_{2}$ & $b_{3}$ & $J_{n}$ \\
\hline 1 & 1 & 0.97172 & -0.71945 & -0.69868 & 0.47786 & 1.0 & 0.17364 & -0.74699 & 0.07901 & 2.73850 \\
\hline 1 & 2 & 0.90883 & -2.68420 & 2.58361 & -0.81651 & 1.0 & -1.73551 & 0.52496 & 0.21039 & 2.63784 \\
\hline 2 & 1 & -0.86396 & 5.12498 & 0.02425 & -3.24367 & 1.0 & 2.82189 & 2.65523 & 0.83178 & 0.69388 \\
\hline 2 & 2 & -1.67377 & 1.90666 & 18.66101 & -18.44336 & 1.0 & 2.78033 & 2.56945 & 0.78788 & 3.56635 \\
\hline
\end{tabular}

\section{Conclusions}

The recent advances in fractional calculus point towards important developments in the application of this mathematical concept. During the last years several algorithms for the approximate calculation of fractional derivatives and integrals were proposed, namely based on Padé fraction expansions. Nevertheless, the resulting expressions are non-optimal revealing the approximation should be formulated as an optimization problem. In this paper a new method, based on evolutionary concepts, for the calculation of fractional expressions, was proposed. In this line of thought, two alternative fitness functions and two possible frequency sampling sets were introduced for the optimization through genetic algorithms. The results demonstrate the excellent performance and the adaptability to different types of expressions.

\section{References}

[1] Oldham KB, Spanier J. The fractional calculus: theory and application of differentiation and integration to arbitrary order. Academic Press; 1974.

[2] Samko SG, Kilbas AA, Marichev OI. Fractional integrals and derivatives: theory and applications. Gordon and Breach Science Publishers; 1993.

[3] Miller KS, Ross B. An introduction to the fractional calculus and fractional differential equations. John Wiley and Sons; 1993.

[4] Podlubny I. Fractional differential equations. San Diego: Academic Press; 1999.

[5] Bagley RL, Torvik PJ. Fractional calculus - a different approach to the analysis of viscoelastically damped structures. AIAA J 1983;21:741-8.

[6] Oustaloup A. La commande CRONE: commande robuste d'ordre non entier. Éditions Hermès, Paris, 1991. ISBN 2-86601-289-5, ISSN 0989-3571.

[7] Anastasio TJ. The fractional-order dynamics of brainstem vestibulo-oculomotor neurons. Biol Cybernet 1994;72(1):69-79.

[8] Mainardi F. Fractional relaxation-oscillation and fractional diffusion-wave phenomena. Chaos Soliton Fract 1996;7:1461-77.

[9] Machado JT. Analysis and design of fractional-order digital control systems. J Syst Anal Model Simulat 1997;27:107-22.

[10] Nigmatullin R. The statistics of the fractional moments: is there any chance to "read quantitatively" any randomness? Signal Proces 2006;86(10):2529-47.

[11] Tarasov VE, Zaslavsky GM. Fractional dynamics of systems with long-range interaction. Commun Nonlinear Sci Numer Simul 2006;11(8):885-98.

[12] Sabatier J, Agrawal OP, Tenreiro Machado JA, editors. Advances in fractional calculus. Theoretical developments and applications in physics and engineering. Springer; 2007, ISBN 978-1-4020-6041-0.

[13] Hedrih K. Vibration modes of an axially moving double belt system with creep layer. J Vib Control 2008;14(9/10):1333-47.

[14] Baleanu D. About fractional quantization and fractional variational principles. Commun Nonlinear Sci Numer Simul $2009 ; 14(6): 2520-3$.

[15] Podlubny I. Fractional-order systems and $\mathrm{PI}^{k} \mathrm{D}^{l}$-controllers. IEEE Trans Automat Control 1999;44(1):208-13.

[16] Machado JT. Discrete-time fractional-order controllers. J Fract Calculus Appl Anal 2001;4:47-66.

[17] Chen YQ, Moore KL. Discretization schemes for fractional-order differentiators and integrators. IEEE Trans Circuit Syst I: Fundam Theory Appl 2002;49(3):363-7.

[18] Tseng CC. Design of fractional order digital fir differentiators. IEEE Signal Process Lett 2001;8(3):77-9.

[19] Vinagre BM, Chen YQ, Petras I. Two direct Tustin discretization methods for fractional-order differentiator/integrator. J Franklin Inst 2003;340(5):349-62.

[20] Chen YQ, Vinagre BM. A new IIR-type digital fractional order differentiator. Signal Proces 2003;83(11):2359-65.

[21] Barbosa R, Machado J, Silva M. Time domain design of fractional differintegrators using least squares approximations. Signal Proces 2006;86(10):2567-81. 
[22] Al-Alaoui MA. Novel digital integrator and differentiator. Electron Lett 1993;29(4):376-8.

[23] Holland JH. Adaptation in natural and artificial systems. Ann Arbor: University of Michigan Press; 1975.

[24] Goldenberg DE. Genetic algorithms in search optimization, and machine learning. Reading, MA: Addison-Wesley; 1989.

[25] Machado JT. Calculation of fractional derivatives of noisy data with genetic algorithms. Nonlinear Dynam. doi:10.1007/s11071-008-9436-1. 